\title{
O Processo de Ensino e Aprendizagem do Raciocínio Clínico pelos Estudantes de Medicina da Universidade Estadual de Londrina
}

\author{
The Teaching and Learning Process in \\ Clinical Reasoning Among Medical Students \\ at the State University in Londrina, Paraná
}

Célia Cristina Fornaziero

Pedro Alejandro Gordan ${ }^{I}$ Mara Lúcia Garanhani

\section{PALAVRAS-CHAVE: \\ - Diagnóstico \\ - Resolução de Problemas \\ - Métodos \\ - Aprendizagem \\ - Educação Médica \\ - Internato e Residência}

Recebido em: 16/07/2010

Reencaminhado em: 04/12/2010

Aprovado em: 04/02/2011

\section{ABSTRACT}

One of the most relevant current issues in teaching, and which has a major impact on clinical practice, is the way physicians elaborate their clinical reasoning. This study focused on understanding the reasoning process developed by medical students at the State University in Londrina, Paraná State, Brazil, identifying the teaching-learning process in clinical reasoning, its conceptualization, and the difficulties that emerge in the related learning process. This was a qualitative study in which the sample consisted of 16 sixth-year medical students (interns). The participants were interviewed on the conceptualization and teaching-learning process in clinical reasoning, and the results were evaluated with discourse analysis. The teaching-learning process in clinical reasoning deals with aspects reported by the students: the professor as a model professional, the importance of communication in clinical care, and conditions that favor learning. The grasp of learning in clinical reasoning encompassed the difficulties and the meanings of clinical reasoning expressed by the interviewees. The qualitative analysis allowed obtaining data that can foster a better understanding of the clinical reasoning process. 


\section{INTRODUÇÃO}

Um dos grandes desafios de uma universidade, na contemporaneidade, permanece o de formar pessoas criativas frente às complexidades do mundo. Esse profissional, idealmente, deve conhecer a tecnologia em vigência, dominar dinâmica de grupo, ser uma pessoa sem preconceitos e também ser capaz de lidar com o pluralismo social ${ }^{1}$.

Atualmente, os educadores concordam em que, para que ocorra uma formação integral do aluno, deve ser utilizada uma pedagogia interativa, que priorize estratégias ativas de ensino-aprendizagem ${ }^{2}$.

Um exemplo de projeto político-pedagógico que considera o contexto social, econômico e cultural da comunidade, além da realidade epidemiológica e dos serviços de saúde, com formação integral do aluno, é o curso de Medicina da Universidade Estadual de Londrina (UEL) ${ }^{3}$. Esse curso visa a uma formação crítica dos estudantes, com o desenvolvimento de competência técnica, enfatizando o núcleo escola/comunidade, embasando o exercício da medicina sobre alicerces científicos, com concepção profissional voltada ao modelo de saúde vigente no Brasil ${ }^{4}$.

A inserção das metodologias ativas no processo de ensino-aprendizagem gerou uma reflexão sobre temas relacionados ao desenvolvimento cognitivo do estudante. O ponto que norteou este artigo foi compreender a percepção que os estudantes de Medicina têm do seu aprendizado em relação ao processo de raciocínio clínico.

Neste contexto educacional, esta pesquisa teve como intuito compreender o processo de ensino e aprendizagem do raciocínio clínico desenvolvido por estudantes do curso de Medicina da UEL, identificando o desenvolvimento desse processo, o papel do professor, a importância da comunicação no atendimento ao paciente e as condições favoráveis ao aprendizado, bem como a concepção de raciocínio clínico e as dificuldades que emergem de seu processo de aprendizagem.

\section{METODOLOGIA}

Esta pesquisa foi realizada no Centro de Ciências da Saúde da UEL, abrangendo internos do curso de Medicina, fundamentado na metodologia da aprendizagem baseada em problemas. O internato médico é a última fase da graduação, na qual o estudante recebe orientação teórica e prática em estágios supervisionados com 24 meses de duração, a partir da quinta série.

Trata-se de um experimento conduzido no primeiro semestre de 2009 para alcançar resultados quantitativos e qualitativos a partir de experiências dos internos do curso de Medicina. A amostra foi formada por 16 internos do início do sexto ano. O número de participantes não foi definido a priori, mas, após as entrevistas com os 16 participantes, observou-se a presença de convergências suficientes para analisar o fenômeno investigado, alcançando a saturação dos dados 5 .

Todos os internos foram convidados a participar via $e$ -mail. Os primeiros de cada grupo de estágio que nos responderam foram contatados por telefone para o agendamento dos encontros. A amostra abrangeu casualmente oito internos do sexo masculino e oito do sexo feminino. O único critério de exclusão foi já ter sido contatado outro interno do mesmo grupo.

Todos os participantes assinaram o termo de consentimento livre e esclarecido para participarem do estudo. O projeto foi aprovado pelo Comitê de Ética do Hospital Universitário da UEL, parecer CEP nº 195/2008.

Foi preparada uma sequência de dez casos clínicos, cada um dos quais continha um breve relato, incluindo sintomas presentes, história pregressa e dados laboratoriais do paciente. Os temas abordados, segundo a realidade epidemiológica do Estado do Paraná, estavam incluídos na grade curricular do curso de Medicina da UEL.

Os casos foram categorizados em dois tipos por três especialistas: cinco foram considerados fáceis (colecistopatia, mononucleose infecciosa, lúpus eritematoso sistêmico, hipotireoidismo e pneumonia) e outros cinco considerados difíceis (insuficiência cardíaca congestiva, endocardite bacteriana, tuberculose pulmonar, nefropatia diabética e síndrome da imunodeficiência adquirida). A concordância diagnóstica final obtida entre os especialistas foi de $96 \%$. As diferenças foram discutidas entre os clínicos, e foi estabelecido um diagnóstico final para todos os casos.

As entrevistas semiestruturadas ${ }^{6}$ foram consideradas uma oportunidade de interação entre a pesquisadora e os participantes do estudo, estratégia adequada para trabalhar o tema da concepção de raciocínio clínico e a percepção dos internos em relação a sua prática clínica, bem como evidenciar questões referentes ao ambiente de aprendizagem do estudante.

Um estudo piloto foi aplicado a quatro estudantes de Medicina da UEL, que não participaram da pesquisa. As questões desenvolvidas durante as entrevistas foram: Como você aprendeu a raciocinar clinicamente? O que é raciocínio clínico para você?

Os encontros foram agendados, e cada sessão foi dividida em duas partes. Na primeira, o interno resolveu, individualmente, a sequência de casos clínicos, apresentados em um notebook com webcam para a gravação deste processo. Os casos foram mostrados aos participantes do estudo alternando-se a ordem de complexidade dos mesmos, sendo apresentado um caso por página. 
A página inicial continha instruções sobre como os internos deveriam proceder. Eles foram orientados a verbalizar todo o pensamento durante a resolução dos casos. Não foi permitido retornar ao caso anterior ou pedir explicações sobre os casos durante o processo de resolução, e o tempo não foi limitado.

Na segunda parte do encontro, após a resolução dos casos, os internos foram entrevistados pela pesquisadora principal. Todas as entrevistas foram transcritas, para análise das falas dos participantes do estudo.

O corpus desta pesquisa incluiu os vídeos de resolução dos casos clínicos, as entrevistas transcritas e as observações da pesquisadora, registradas em um diário de campo durante todo o encontro com os internos. Na interpretação das entrevistas, foi utilizada a análise de discurso pela abordagem da Estrutura do Fenômeno Situado ${ }^{7}$, constituída por dois momentos: análise individual ou ideográfica e análise geral.

A análise individual engloba leituras flutuantes das falas dos entrevistados, sem buscar ainda qualquer interpretação. O objetivo dessas leituras foi a familiarização da pesquisadora com o conteúdo dos discursos. Após as leituras, foram discriminadas as unidades de significado (palavras ou frases que possuem algum sentido na perspectiva dos objetivos da pesquisa). Em seguida, foram feitas as interpretações e convergências das unidades de significado dentro do discurso de cada entrevistado.

No segundo momento, foi realizada a análise geral, que consiste na compreensão e articulação dos diversos casos individuais como exemplos particulares de algo mais comum dentro da amostra. Nesta etapa, ocorreu a releitura das entrevistas, para perceber as unidades de significado interpretadas de cada caso como pertencentes ao todo do fenômeno estudado.

Em seguida, foi realizada a aproximação destas unidades, identificando convergências e divergências presentes entre os internos, possibilitando a construção dos temas da prática de raciocínio clínico que configuraram a estrutura do fenômeno estudado. Apesar de se ter, didaticamente, dividido cada tópico temático, trata-se de um universo indissolúvel e temporalmente inseparável.

As imagens de vídeo foram editadas e observadas por dois pesquisadores, que fizeram apontamentos a respeito da prática de resolução dos casos clínicos. Para complementar as interpretações realizadas, foram adicionadas às análises as percepções registradas no diário de campo pela pesquisadora.

Desta forma, o estudo abrangeu imagens de vídeo da resolução de casos clínicos, entrevistas e registro no diário de campo. Em particular, a análise das entrevistas possibilitou identificar a concepção e a percepção do processo de ensino e aprendizagem do raciocínio clínico pelos participantes do estudo.

A metodologia descrita é parte de uma tese de doutorado em Ciências da Saúde. Este artigo foi construído a partir dos resultados obtidos sobre o processo de ensino-aprendizagem do raciocínio clínico e sua apreensão por parte dos estudantes.

\section{RESULTADOS E DISCUSSÃO}

O processo de ensino e aprendizagem do raciocínio clínico trata dos aspectos relatados pelos estudantes quanto ao aprendizado do raciocínio clínico: o docente como modelo a seguir, a importância da comunicação no atendimento clínico e as condições favoráveis ao aprendizado. Em um segundo momento, ficou ilustrada a concepção de raciocínio clínico e as dificuldades que emergem de seu processo de aprendizagem.

\section{Docente como Modelo Profissional}

Em relação ao processo de aprendizagem, os internos assinalaram o docente como modelo do conhecimento. Dentre as diversas formas de aprender apresentadas - por repetição, leitura ou estudos individuais - , o professor foi categoricamente destacado como modelo a ser seguido pelo estudante, independentemente do período do curso - tutorial, aulas de habilidades ou internato.

\footnotetext{
"Acho que eu copiei isso dos docentes, como eles pensavam, como eles raciocinavam. Acho que eu fui copiando. Foi assim que eu aprendi." (interno 16)
}

A eficácia do professor é um atributo essencial ao trabalho docente, considerando-se que o estudante o observa como modelo profissional. Assim, os programas de desenvolvimento docente deveriam almejar fortalecer não apenas as habilidades pedagógicas, mas também as habilidades afetivas, tanto para professores experientes quanto para novatos. Os professores devem ter suporte da instituição onde trabalham ${ }^{8}$.

Um importante fator de interferência na formação profissional é a motivação. Estudiosos ${ }^{9,10}$ afirmam que o sucesso das estratégias de ensino depende da motivação do professor. A motivação intrínseca do professor tem papel de destaque nas práticas instrucionais e na ampliação da motivação dos estudantes. Os alunos também se sentem apoiados por professores menos autoritários e mais cooperativos, e o entusiasmo dos docentes pode criar um ambiente estimulador ao uso de vários métodos de ensino ${ }^{11}$.

Em nossa pesquisa, o professor requerido, durante o tutorial, foi aquele que orientava a observação dos fatos e auxiliava o aluno a enfrentar o medo do desconhecido. 
"Acho que foi nos primeiros tutoriais, ajudava quando o tutor estava participando, ajudava bastante a gente a olhar e observar as coisas sem medo. Então, eu acho que ter um bom tutor, um bom tutorial ajuda." (interno 3)

O grupo tutorial pode ser considerado um meio para associar conhecimento cognitivo e sua aplicação em problemas clínicos no início da formação médica ${ }^{12}$. Além disso, tem o papel de promover a ativação de conhecimento prévio, mudar conceitos e colaborar para a construção de conhecimento, mas isto só ocorre com o envolvimento do tutor. Usar a experiência pessoal dos estudantes na prática clínica nos problemas do grupo pode conduzi-los a uma melhor interpretação individual dos problemas ${ }^{13}$.

Sendo conduzido segundo essas premissas pelo professor, o grupo tutorial objetiva preparar o aluno para realizar o raciocínio clínico com maior segurança, já que o estudante pode vivenciar discussões sobre casos clínicos encontrados na rotina ambulatorial.

Os internos também relataram que as aulas de habilidades oferecem diferentes oportunidades de interação professor-aluno. No entanto, solicitaram maior participação do professor orientador, com explicações técnicas no ambulatório.

"Embora os professores sejam bem intencionados, ainda falta isso na medicina! Ah, você tem que seguir esse e aquele caminho, eu não achei que ficou bem explicado, faltaram experiências boas que me orientassem." (interno 14)

Os resultados de nossa pesquisa possibilitam refletir sobre as situações pedagógicas que estão sendo utilizadas nas aulas de habilidades para motivar o aprendizado dos alunos. Pelo discurso dos internos, podemos inferir o desejo de vivenciar atividades teóricas e práticas reais, com discussão no contexto clínico, principalmente durante as aulas de habilidades.

O desenvolvimento de habilidades, valores e atitudes deveria ser ressaltado pelo menos na mesma extensão da aquisição de conhecimentos. Isto sem esquecer que a habilidade de cuidar dos pacientes e resolver seus problemas é considerada por muitos educadores como o maior objetivo da educação médica ${ }^{14}$.

O conhecimento clínico dos médicos é revisado e estendido continuamente por meio de numerosas interações com o paciente, resultando em um raciocínio clínico eficiente e adequado $^{15}$.

A prática clínica não é uma entidade separada da educação e deve estar direcionada ao processo cognitivo, que tem grande impacto na decisão diagnóstica ${ }^{16}$.
Planejar o ensino com o intuito de criar ambientes de aprendizagem e experiências apropriadas aos estudantes requer ter ciência do que eles mesmos percebem como importante em seu desenvolvimento pessoal e profissional durante sua graduação ${ }^{17}$.

O tema destacado pelos entrevistados ao abordarem o internato foi a discussão de casos clínicos de forma direcionada pelos docentes, mostrando como agir para desenvolver o raciocínio clínico e continuar o procedimento na prática. A figura de um professor companheiro se mostrou capaz de promover maior interação entre o estudante e o paciente.

“E mesmo agora no internato, há uns docentes que, quando vão discutir um caso, separam por partes: primeiro a história, tentar levantar um raciocínio em cima da história, para depois você continuar." (interno 15)

Um grupo de pesquisadores ${ }^{18}$ reforça que, durante o internato, deveria ser despendido mais tempo para possibilitar reflexões entre pares e com os médicos orientadores.

Os professores clínicos deveriam promover o desenvolvimento do raciocínio e, simultaneamente, fazer o diagnóstico do problema do paciente e das habilidades do estudante, e, desta forma, assegurar a qualidade do cuidado ao paciente e imprimir profissionalismo enquanto promove a habilidade de raciocínio. Este conjunto de ações constitui a arte do professor clínico $^{19}$.

\section{A Importância da Comunicação no Atendimento Clínico}

Os internos se mostraram conscientes de suas responsabilidades com os pacientes, desde saber se comunicar até a realização do exame físico.

"Você tem que saber conversar com o paciente, lidar... Saber o que perguntar, o exame deve ser bem feito". (interno 1)

Windish et al. ${ }^{20}$ defendem que ensinar a conexão entre a comunicação com o paciente e o raciocínio clínico permite aos estudantes entender a importância da relação biomédica e dos aspectos psicológicos do cuidado ao paciente.

Além disso, treinar estratégias para focar a aquisição de conhecimento sobre as habilidades de comunicação entre médico e paciente pode reduzir os erros de diagnóstico, pois aumenta o nível de competência do estudante em obter uma história clínica mais detalhada, para conduzi-lo a um exame físico mais informativo ${ }^{20}$.

Para melhorar a comunicação entre o paciente e o médico, é preciso entender a natureza do processo de raciocínio clínico que está sendo considerado na consulta. O médico tem que 
criar uma atmosfera ideal para que o paciente possa expressar tudo o que considere importante ${ }^{21}$.

A ferramenta de diagnóstico mais poderosa disponível é a comunicação. Estima-se que aproximadamente 80\% das informações necessárias ao raciocínio clínico sejam provenientes do paciente. Geralmente, uma pequena parcela de informação que parece irrelevante para o paciente pode fechar o diagnóstico para o médico. Ouvir o paciente é muito importante, mesmo após o diagnóstico ter sido estabelecido ${ }^{22}$.

\section{Condições Favoráveis ao Aprendizado}

Os participantes de nosso estudo também descreveram a necessidade de conhecimento cognitivo sobre as doenças, bem como a existência de condições favoráveis ao aprendizado: boa noite de sono, tranquilidade e disposição para pensar e agir.

“Então, para eu aprender, tenho que estar também com uma boa noite de sono, descansada, bem tranquila, para não ter preguiça de raciocinar." (interno 1 )

A escola médica deve estar preparada e ter em foco esta preocupação, cuidar mais do estudante de Medicina, respeitando-o, escutando-o, ajudando-o a desenvolver estratégias que o preparem para lidar com a pressão que vivenciará no cotidiano acadêmico e profissional, bem como dando suporte psicológico e pedagógico de forma geral e em especial àqueles que passam por dificuldades ${ }^{23}$.

O bem-estar do estudante pode ser considerado o maior indicador da qualidade de educação ${ }^{24}$. Desse modo, as relações interpessoais entre docentes e estudantes são um importante aspecto do desenvolvimento das aulas.

Independentemente do currículo empregado e das metodologias aplicadas durante o curso, os responsáveis por mudanças pedagógicas deveriam considerar que o estudante e o professor, não o currículo, são os elementos cruciais em um programa educacional ${ }^{25}$.

Infelizmente, o aumento do número de alunos originou uma defasagem entre a demanda estudantil e a oferta de recursos institucionais, que, em muitos casos, não estavam sendo mais adequados às necessidades formativas e informativas que os futuros profissionais requeriam durante seus estudos de graduação ${ }^{26}$.

\section{Os Significados do Raciocínio Clínico para os Estudantes}

Quanto à apropriação do aprendizado do raciocínio clínico, os entrevistados expressaram os significados de raciocínio clínico. A assimilação de conteúdo pode ser analisada pelos conceitos que os sujeitos imprimem a respeito do assunto.
Para os sujeitos da pesquisa, o raciocínio clínico foi contextualizado como: processo, isto é, etapas progressivas que exigem esforço para a construção do saber; quebra-cabeça que demanda organização para selecionar e juntar todas as partes a fim de obter êxito; desvendar a resposta inserida no contexto do paciente; arquitetar um plano na cabeça com o fim de atingir o alvo desejado; e a conduta frente ao caso, ou seja, a definição de uma estratégia terapêutica para minimizar a chance de erros.

\begin{abstract}
Processo
"São as etapas que você segue para chegar a um bom diagnóstico, uma hipótese diagnóstica. Raciocínio clínico é algo progressivo, desde o primeiro contato com o paciente até os exames, porque os exames diagnósticos laboratoriais ajudam no raciocínio, mas é algo progressivo."(interno 1)
\end{abstract}

\section{Quebra-cabeça}

"Mas acho que raciocínio clínico é mais ou menos isso, você tentar juntar o quebra-cabeça e ver a coisa mais palpável." (interno 13)

\section{Desvendar a resposta}

"Raciocínio seria conseguir enxergar quais são os dados relevantes na história e no exame físico do paciente." (interno 5)

\section{Arquitetar um plano \\ "Raciocínio clínico seria ter um plano na cabeça, uma se- quência." (interno 12) \\ Conduta frente ao paciente \\ "Raciocínio não é somente chegar a um diagnóstico. Raciocí- nio é conseguir encaminhar direito, porque nem sempre você vai ter um diagnóstico. Você precisa resolver o problema da- quele paciente, confortá-lo." (interno 6)}

Embora os significados expressos se relacionem com a concepção de raciocínio clínico, a diferença mostrada reside na forma de compreensão das partes, ora de maneira separada, ora de maneira articulada. Alguns internos percebem o raciocínio clínico parcialmente, como um fragmento de uma parte isolada de um todo.

Os internos citam o quebra-cabeça, mas não conseguem visualizar a imagem final construída. Mencionaram busca de respostas, planejamento de ações e de condutas, mas, por vezes, não conseguiam articular estas ações como etapas de um processo único, o raciocinar. Todavia, alguns internos apresentaram uma concepção mais integral, visualizando o raciocínio clínico como um processo dinâmico que envolve várias partes articuladas entre si. 
Os autores são unânimes em considerar o raciocínio clínico como um processo. Diagnosticar começa com a geração de uma hipótese diagnóstica inicial por um processo de informação automático. O processo de raciocínio pode parar se a hipótese for aceita, ou um processo analítico pode ser implantado para refinar hipóteses ${ }^{27-30}$.

\section{Dificuldades Vivenciadas na Aprendizagem do Raciocínio Clínico e Alternativas Possíveis}

Quando questionados sobre seus conceitos de raciocínio clínico, os internos relataram suas dificuldades em exercer este processo. O grande problema que emergiu nesta área foi a incerteza de saber se o que está sendo aplicado na rotina clínica está correto ou não.

"Eu acho difícil dizer como se aprendeu a fazer diagnóstico. Eu acho que até hoje eu não sei fazer." (interno 5)

Este quadro de incerteza pode ser analisado à luz da aprendizagem significativa ${ }^{31}$, segundo a qual a aprendizagem se estrutura complexamente, em um movimento de continuidade/ruptura. O processo de continuidade é aquele em que o estudante é capaz de relacionar o conteúdo apreendido aos conhecimentos prévios, ou seja, o conteúdo novo deve se apoiar em estruturas cognitivas existentes e organizadas. Já o processo de ruptura se instaura a partir do surgimento de novos desafios, que deverão ser trabalhados pela análise crítica, levando o aprendiz a ultrapassar suas vivências - conceitos prévios, sínteses anteriores e outros - , tensão que permite ampliar suas possibilidades de conhecimento ${ }^{31}$.

Em relação ao contexto de aprendizado, um grupo de estudiosos $^{32}$ sugere que deveria ser ministrado um curso para oportunizar o processo de raciocínio clínico com o emprego de problemas específicos e exemplos, antes de encontrar pacientes reais, pois as dificuldades percebidas pelos estudantes, em sua pesquisa, residiam em como conseguir os dados, interpretar e escolher os mais relevantes, sintetizar a informação e organizá-la hierarquicamente. Concordamos em que este tipo de estratégia pode minimizar a insegurança do estudante em seu processo de aprendizagem.

Uma perspectiva a considerar no processo de ensino-aprendizagem é a necessidade de entender como as pessoas decidem se o que elas sabem é suficiente ou se é preciso estudar mais. Para tanto, os educadores da área da saúde têm que desenvolver atividades de feedback rotineiramente ${ }^{33}$. Embora os internos não tenham falado explicitamente sobre o feedback, ficou clara a necessidade de abordar esta técnica de ensino, visto que vários participantes alertaram para a dificuldade de orientação e confirmação sobre o que estavam realmente aprendendo.

\footnotetext{
"Eu acho que não aprendi tão bem a raciocinar clinicamente. Não sei se eu aprendi completamente. É uma coisa meio difícil, não sei direito." (interno 2)
}

Dar e receber feedback são atitudes críticas que os professores deveriam ensinar desde o início do processo de educação médica ${ }^{34}$. Aprender efetivamente com o feedback requer retornar o conteúdo de maneira que quem o recebe possa ouvi-lo, refletir sobre ele e considerá-lo em suas decisões. Por isso, é essencial que esse processo respeite sempre os pares e a diferença de níveis de conhecimento ${ }^{35}$.

Estudantes e docentes constituem o binômio fundamental do processo ensino-aprendizagem. As percepções e sugestões de um e de outro se tornam relevantes nos momentos de introduzir mudanças para melhorar a qualidade do ensino. A necessidade de incrementar as atividades práticas nas escolas de Medicina é reconhecida e deve estar de acordo com o perfil do profissional médico que as faculdades definiram em seus currículos ${ }^{26}$.

Destacamos a responsabilidade presente e futura daqueles que devem planificar um currículo flexível para a medicina, levando em conta as diversas fontes de informação docente e estudantil que vêm assinalando a necessidade de incrementar a prática na carreira médica ${ }^{26}$.

Alguns pesquisadores ${ }^{36}$ alertam que a educação médica está, como o serviço de saúde que a suporta, indo rápido e, em alguns casos, com mudanças radicais. Estas alterações refletem pensamentos sobre os caminhos a apresentar à educação. O problema é que estes pensamentos, frequentemente, são alicerçados em desarticulação com o modelo tradicional de educação e, muitas vezes, elaborados por uma equipe sem compromisso com a qualidade e com a eficiência.

A educação médica deve ser vista como um processo social e de transformação pessoal, resultando em estudantes e clínicos capazes de cuidar de seus pacientes e resolver seus problemas com ativo envolvimento pessoal ${ }^{36}$.

\section{CONSIDERAÇÕES FINAIS}

Quando abordamos o sentido de raciocinar clinicamente, vemos o esboço de um processo mental formado por etapas progressivas que contribuem para a construção do saber e conduzem ao entendimento de determinado assunto ou de um caso clínico, culminando com atitudes efetivas para a transformação da realidade que gerou esse processo de reflexão.

Esta abordagem é importante porque define que o processo de raciocinar depende da formação de uma rede semântica 
do conhecimento adquirido por seu interlocutor. Todo processo deve ser gerado na mente e norteado pelos conhecimentos adquiridos pelo médico durante sua formação profissional.

Em contrapartida, a formação profissional deve considerar as expectativas e percepções dos estudantes, principalmente em relação ao papel do professor, que imprime grande influência na condução de todo o processo educacional.

O professor apresentado como modelo profissional fortalece a necessidade de refletir sobre o papel do docente no projeto político-pedagógico de um curso. Motivação, articulação da comunicação no atendimento ao paciente e atuação como agente de condições favoráveis ao aprendizado são algumas atitudes que mostram que o professor sempre foi e continua sendo um componente fundamental do processo de aprendizagem dos estudantes.

Ainda há muito a explorar no processo de formação profissional em saúde, e este estudo enfatiza a importância de refletir sobre temas essenciais ao desenvolvimento do estudante de Medicina. Todos aqueles que estão envolvidos com o processo de ensino-aprendizagem deveriam considerar esses temas, com o propósito de minimizar as dificuldades de obtenção de uma formação mais integral.

\section{REFERÊNCIAS}

1. Fornaziero CC, Gil CR. Novas tecnologias aplicadas ao ensino da anatomia humana. Rev Bras Educ Med. 2003; 27:141-6.

2. Contribuição para as novas diretrizes curriculares dos cursos de graduação da área de saúde. Olho Mágico [periódico na internet]. 1998 [acesso em 3 abr. 2010];4. Disponível em: http:/ / www.revistaolhomagico.com.br.

3. Feuerwerker LCM. Além do discurso de mudança na educação médica: processo e resultados. São Paulo: Hucitec; 2002.

4. Universidade Estadual de Londrina. Projeto Político Pedagógico Institucional da UEL. Londrina, 2002 [online]. [acesso em 3 abr. 2010]. Disponível em: http://www.uel. $\mathrm{br} / \mathrm{cae} /$.

5. Fontanella BJB, Ricas J, Turato ER. Amostragem por saturação em pesquisas qualitativas em saúde: contribuições teóricas. Cad Saúde Pública. 2008;24:17-27.

6. Duarte R. Pesquisa Qualitativa: reflexões sobre o campo de trabalho. Cad Pesq.2002; 115:139-54.

7. Martins J, Bicudo MAV. A pesquisa qualitativa em psicologia: fundamentos e recursos básicos. São Paulo: Centauro; 2005.

8. Yeo LS, Ang RP, Chong WH, Huan VS, Quek CL. Teacher efficacy in the context of teaching low achieving students. Curr Psychol. 2008;27:192-204.
9. Muller $C$. The minimum competency exam requirement teachers' and students' expectations and academic performance. Soc Psych of Educ. 1997;2:199-216.

10. Lam SF, Cheng RW, Ma WYK. Teacher and student intrinsic motivation in project-based learning. Instr Sci. 2009;37:565-78.

11. Petegem KV, Aelterman A, Keer HV, Rossel Y. The influence of student characteristics and Interpersonal teacher behavior in the classroom on student's wellbeing. Soc Indic Res. 2008;85:279-91.

12. Dolmans D, Schmidt HG. What do we know about Cognitive and Motivational Effects of Small Group Tutorials in Problem-Based Learning? Adv in Health Sci Educ. 2006; 11:15.

13. Mamede S, Schmidt HG, Norman GR. Innovations in Problem-based Learning: What can we Learn from Recent Studies? Adv in Health Sci Educ. 2006;11:403-22.

14. Schmidt HG, Dauphinee DW, Patel VL. Comparing the Effects of Problem-Based and Conventional Curricula in an International Sample. Acad Med. 1987;62:305-15.

15. Bruin ABH, Schmidt HG, Rikers RMJ. The role of basic science knowledge and clinical knowledge in diagnostic reasoning: a structural equation modeling approach. Acad Med. 2005;80:765-72.

16. Dunphy BC, Cantwell R, Bourke S, Fleming M, Smith B, Joseph KS, Dunphy SL. Cognitive elements in clinical decision-making toward a cognitive model for medical education and understanding clinical reasoning. Adv in Health Sci Educ. 2009;14.

17. Lown N, Davies I, Cordingley L, Bundy C, Braidman I. Development of a method of investigate medical students' perceptions of their personal and professional development. Adv in Health Sci Educ. 2009;14:475-86.

18. Schmidt HG, Rikers RMJP. How expertise develops in medicine: knowledge encapsulation and illness script formation. Med Educ. 2007;41:1133-9.

19. Bowen JL. Medical Education: Educational Strategies to Promote Clinical Diagnostic Reasoning. N Engl J Med. 2006;355:2217-25.

20. Windish DM, Price EG, Clever SL, Magaziner JL, Thomas PA. Teaching medical students the important connection between communication and clinical reasoning. J Gen Intern Med. 2005;20:1108-13.

21. Charles C, Gafni A, Whelan T. How to improve communication between doctors and patients: Learning more about the decision making context is important. BMJ. 2000; 320:1220-1.

22. O'Dowd GVG. Doctor-Patient Communication: An Introduction for Medical Students. Journal of Hamamatsu University School of Medicine. 2004;18:1-14. 
23. Zonta R, Robles AC, Grosseman S. Estratégias de enfrentamento do estresse desenvolvidas por estudantes de Medicina da Universidade de Santa Catarina. Rev Bras Educ Med. 2006; 30:147-53.

24. Petegem KV, Aelterman A, Keer HV, Rosseel Y. The influence of student characteristics and Interpersonal teacher behavior in the classroom on student's wellbeing. Soc Indic Res. 2008;85:279-91.

25. Clough RW, Lehr R P. Testing Knowledge of Human Gross Anatomy in Medical School: An Applied Contextual-Learning Theory Method. Clin Anat. 1996;9:5.

26. Barrovecchio JCP, Paz LB. Sugerencias acerca del proceso de ensenãnza-aprendizaje en anatomía humana. Rev Chil Anat. 1998;16:5.

27. Eva KW. What every teacher needs to know about clinical reasoning. Med Educ. 2004; 39:98-106.

28. Mamede S. Schmidt HG, Rikers RMJP, Penaforte JC, Coelho-Filho JM. Breaking down automaticity: case ambiguity and the shift to reflective approaches in clinical reasoning. Med Educ. 2007;41:1185-92.

29. Norman GR, Young M, Brooks L. Non-analytical models of clinical reasoning: the role of experience. Med Educ. 2007;41:1140-5.

30. McLaughlin K, Heemskerk L, Herman R, Ainslie M, Rikers RM, Schmidt HG. Initial diagnostic hypotheses bias analytic information processing in non-visual domains. Med Educ. 2008;42:496-502.

31. Ausubel D, Novak JD, Hanesian H. Educational Psychology, a Cognitive View. New York: Holt, Reinhart and Winston; 1978

32. Gessel EV, Nendaz MR, Vermeulen B, Funod A, Vu NV. Development of clinical reasoning from the basic sciences to the clerkships: a longitudinal assessment of medical stu- dents' needs and self-perception after a transitional learning unit. Med Educ. 2003;37:966-74.

33. Eva WK. Diagnostic error in medical education: where wrongs can make rights. Adv in Health Sci Educ. 2009;14(supl. 1):71-81.

34. Kruidering-Hall M, O'Sullivan PS, Chou CL. Teaching Feedback to First-year Medical Students: Long-term Skill Retention and Accuracy of Student Self-assessment. JGIM. 2009;24:721-6.

35. Henderson P, Ferguson-Smith AC, Johnson, MH. Developing essential professional skills: a framework for teaching and learning about feedback. BMC Med Educ. 2005;5: 11-6.

36. McLachlan JC, Bligh J, Bradley P, Searle J. Teaching Anatomy without cadavers. Med Educ. 2004;38:6.

\section{CONTRIBUIÇÃO DOS AUTORES}

Célia Cristina Fornazieiro autora do projeto de pesquisa, coleta e análise de dados, redação do texto. Pedro Alejandro Gordan orientação e avaliação do projeto de pesquisa, bem como participante da análise dos dados coletados, auxiliou na redação do texto final. Mara Lúcia Garanhani co-orientadora do projeto de pesquisa, análise de dados, orientação das discussões e participou da redação final do texto.

\section{CONFLITO DE INTERESSES}

Declarou não haver.

\section{ENDEREÇO PARA CORRESPONDÊNCIA}

Célia Cristina Fornazieiro

Universidade de Londrina

Campus Universitário - Londrina

CEP. 86051-990 Cx. Postal 6001 — PR

E-mail: celiafornaziero@uel.br 